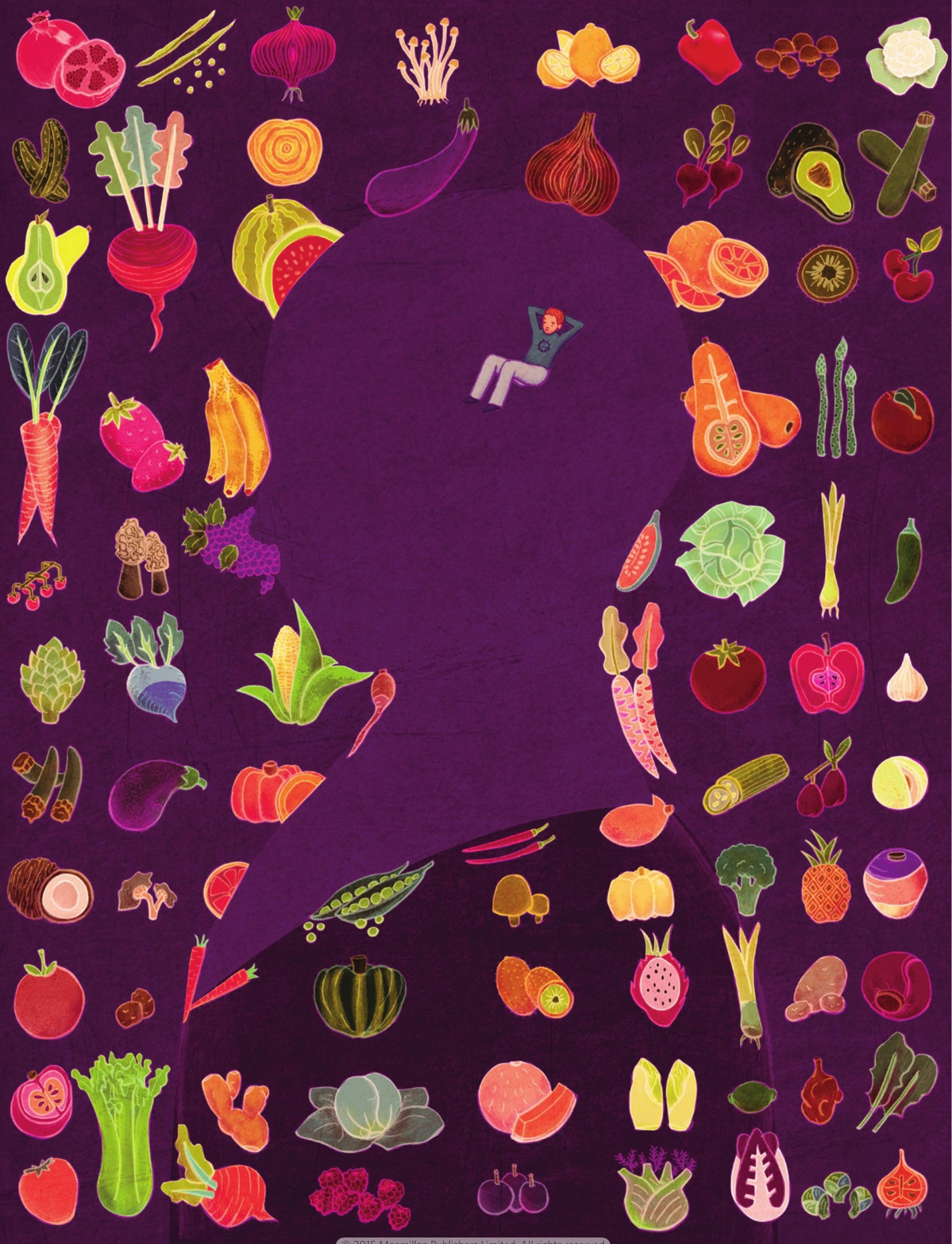


THE NOTION THAT THE STATE of our gut governs our state of mind dates back more than 100 years. Many 19th- and early 20th-century scientists believed that accumulating wastes in the colon triggered a state of "auto-intoxication," whereby poisons emanating from the gut produced infections that were in turn linked with depression, anxiety and psychosis. Patients were treated with colonic purges and even bowel surgeries until these practices were dismissed as quackery.

The ongoing exploration of the human microbiome promises to bring the link between the gut and the brain into clearer focus. Scientists are increasingly convinced that the vast assemblage of microfauna in our intestines may have a major impact on our state of mind. The gut-brain axis seems to be bidirectional-the brain acts on gastrointestinal and immune functions that help to shape the gut's microbial makeup, and gut microbes make neuroactive compounds, including neurotransmitters and metabolites that also act on the brain. These interactions could occur in various ways: microbial compounds communicate via the vagus nerve, which connects the brain and the digestive tract, and microbially derived metabolites interact with the immune system, which maintains its own communication with the brain. Sven Pettersson, a microbiologist at the Karolinska Institute in Stockholm, has recently shown that gut microbes help to control leakage through both the intestinal lining and the blood-brain barrier, which ordinarily protects the brain from potentially harmful agents.

Microbes may have their own evo-

lutionary reasons for communicating with the brain. They need us to be social, says John Cryan, a neuroscientist at University College Cork in Ireland, so that they can spread through the human population. Cryan's research shows that when bred in sterile conditions, germ-free mice lacking in intestinal microbes also lack an ability to recognize other mice with whom they interact. In other studies, disruptions of the microbiome induced mice behavior that mimics human anxiety, depression and even autism. In some cases, scientists restored more normal behavior by treating their test subjects with certain strains of benign bacteria. Nearly all the data so far are limited to mice, but Cryan believes the findings provide fertile ground for developing analogous compounds, which he calls psychobiotics, for humans. "That dietary treatments could be used as either adjunct or sole therapy for mood disorders is not beyond the realm of possibility," he says.

\section{PERSONALITY SHIFTS}

SCIENTISTS USE germ-free mice to study how the lack of a microbiomeor selective dosing with particular bacteria-alters behavior and brain function, "which is something we could never do in people," Cryan says. Entire colonies of germ-free mice are bred and kept in isolation chambers, and the technicians who handle them wear full bodysuits, as if they were in a biohazard facility. As with all mice research, extrapolating results to humans is a big step. That is especially true with germfree mice because their brains and immune systems are underdeveloped, and they tend to be more hyperactive and daring than normal mice.

A decade ago a research team led by Nobuyuki Sudo, now a professor of internal medicine at Kyushu University in Japan, restrained germ-free mice in a narrow tube for up to an hour and then measured their stress hormone output. The amounts detected in the germ-free animals were far higher than those measured in normal control mice exposed to the same restraint. These hormones are released by the hypothalamic-pituitary-adrenal axis, which in the germ-free mice was clearly dysfunctional. But more important, the scientists also found they could induce more normal hormonal responses simply by pretreating the animals with a single microbe: a bacterium called Bifidobacterium infantis. This finding showed for the first time that intestinal microbes could influence stress responses in the brain and hinted at the possibility of using probiotic treatments to affect brain function in beneficial ways. "It really got the field off the ground," says Emeran Mayer, a gastroenterologist and director of the Center for Neurobiology of Stress at the University of California, Los Angeles.

Meanwhile a research team at McMaster University in Ontario led by microbiologist Premsyl Bercik and gastroenterologist Stephen Collins discovered that if they colonized the intestines of one strain of 


\section{The Diverse Microbiome of the Hunter-
Gatherer}

The Hadza of Tanzania offer a snapshot of the co-adaptive capacity of the gut ecosystem

By Stephanie L. Schnorr

We tend to forget that modern humanity is largely sheltered from the last vestiges of wild untamed Earth and that our way of life bears little resemblance to how our ancestors lived during 90 percent of human history. We have lost nearly all trace of these former selvesand, worse, have marginalized the few remaining humans who retain their huntergatherer identity. In Tanzania, tribes of

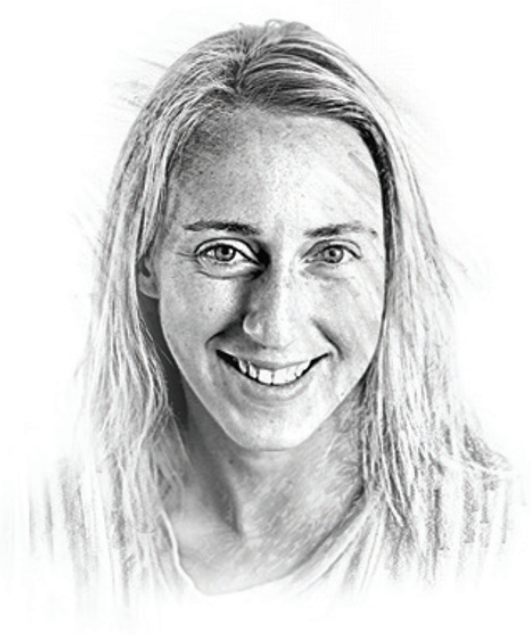

wandering foragers called the Hadza, who have lived for thousands of years in the East African Rift Valley ecosystem, tell us an immense and precious story about how humans, together with their microbial evolutionary partners, are adapted to live and thrive in a complex natural environment.

Ongoing research with the Hadza to characterize the hunter-gatherermicrobiome relationship has yielded not only insight into the co-adaptive capacity of this microbial ecosystem but also a profound appreciation for how versatile human life can be. The microbiome is central to our biology. It mediates the interaction and exchange of information across host-environment thresholds such as the mouth, skin and gut.

The strength and importance of this mediation are borne out in the Hadza gut microbiota. Their microbiome harbors incredibly high taxonomic diversity, indicating great ecosystem stability and flexibility. It is capable of withstanding the perpetual presence of parasites and pathogens and can respond to fluctuations in diet caused by an unpredictable and seasonally dependent food supply. Interestingly, bacterial taxonomic abundance is different in Hadza men and women. Because of the sexual division of labor in Hadza society, men and women tend to consume more of their respective foraged food resources. The women primarily collect and eat tubers and other plant foods. As a result, it appears that women carry more bacteria to help process the plant fiber in their diets. This difference has direct implications for how the gut microbiota may enable Hadza germ-free mice with bacteria taken from the intestines of another mouse strain, the recipient animals would take on aspects of the donor's personality. Naturally timid mice would become more exploratory, whereas more daring mice would become apprehensive and shy. These tendencies suggested that microbial interactions with the brain could induce anxiety and mood disorders.

Bercik and Collins segued into gut-brain research from their initial focus on how the microbiome influences intestinal illnesses. People who suffer from these conditions often have co-occurring psychiatric problems such as anxiety and depression that cannot be fully explained as an emotional reaction to being sick. By colonizing germ-free mice with the bowel contents of people with irritable bowel syndrome, which induces constipation, diarrhea, pain and low-grade inflammation but has no known cause, the McMaster's team reproduced many of the same gastrointestinal symptoms. The animals developed leaky intestines, their immune systems activated, and they produced a barrage of pro-inflammatory metabolites, many with known nervous system effects. Moreover, the mice also displayed anxious behavior, as indicated in a test of their willingness to step down from a short raised platform.

\section{AUTISM CONNECTION?}

SCIENTISTS HAVE ALSO BEGUN to explore the microbiome's potential role in autism. In 2007 the late Paul Patterson, a neuroscientist and developmental biologist at the California Institute of Technology, was intrigued by epidemiological data showing that women who suffer from a high, prolonged fever during pregnancy are up to seven times more likely to have a child with autism. These data suggested an alternative cause for autism besides genetics. To investigate, Patterson induced flulike symptoms in pregnant mice with a viral mimic: an immunostimulant called polyinosinic:polycytidylic acid, or poly(I:C). He called this the maternal immune activation (MIA) model.

The offspring of Patterson's MIA mice displayed all three of the core features of human autism: limited social interactions, a tendency toward repetitive behavior and reduced communication, which he assessed by using a special microphone to measure the length and duration of their ultrasonic vocalizations. In addition, the mice had leaky intestines, which was important because anywhere from 40 to 90 percent of all children with autism suffer from gastrointestinal symptoms.

Then Caltech microbiologist Sarkis Mazmanian and his doctoral student Elaine Hsiao discovered that MIA mice also have abnormal microbiomes. Specifically, two bacterial classes - Clostridia and Bacteroidia - were far more abundant in the MIA offspring than in normal mice. Mazmanian acknowledges that these imbalances may not be the same as those in humans with autism. But the finding was compelling, he says, because it suggested that the behavioral state of the MIA mice - and perhaps by extension autistic behavior in humansmight be rooted in the gut rather than the brain. "That raised a provocative question," Mazmanian says. "If we treated gastrointestinal 
women to obtain adequate nutrition for fertility and reproductive success, despite a resource-limited environment. Through our work with the Hadza, we have been able to contribute to mounting evidence that human microbiota exerts a powerful influence on host health and survival, especially in natural fertility- and subsistence-based populations.

Comparative analysis of the gut microbiota of hunter-gatherers with that of Westernized industrial populations is also beginning to yield important insights. The microbial diversity in industrial groups is far below that of the Hadza, as well as those of other rural farming communities in Burkina Faso, Malawi and South Africa. Whereas a reduction in diversity may not seem ideal, it is the predictable response of an ecosystem facing a narrow range of selective pressures and is therefore no less adaptive. Some technological interventions, such as hypersanitation, consumption of refined foods and habitual use of antibiotics, have had a dramatic impact over time on the functional role of the microbiome in industrial populations. These aspects of a Westernized way of life have to a large extent displaced much of the original mutualistic functions of the microbiome in stabilizing our bodies against foreign microorganisms, allowing us to digest unprocessed foods and helping train our immune system to effectively fight disease.

We are just beginning to understand how the microbiome evolves over our lifetimes as a dynamic and mutualistic ecosystem that helps to facilitate human health. Thanks to the Hadza, we know that ancient human huntergatherers must have maintained a direct and persistent interface with the natural environment. As a result, the ancestral human microbiome was almost certainly a taxonomically diverse community, providing the functional flexibility that accompanied global colonization and is our adaptive legacy.

Stephanie L. Schnorr is a Ph.D. candidate in the Research Group on Plant Foods in Hominin Dietary Ecology at the Max Planck Institute for Evolutionary Anthropology in Leipzig, Germany.
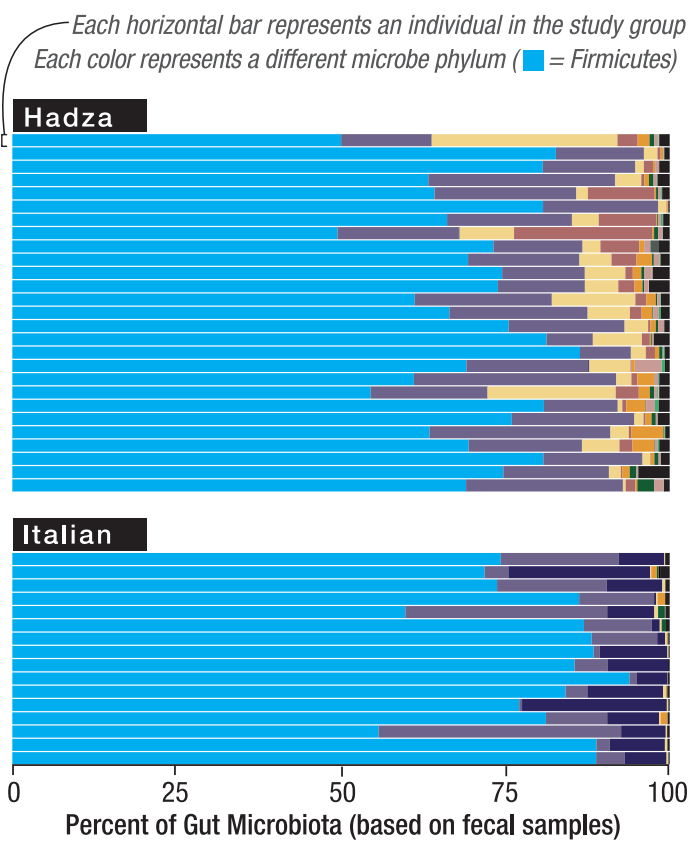

TAXONOMIC TREASURE TROVE:

A survey of fecal microbiota of 43 subjects revealed $a$ more varied mix of gut bacteria phyla among Hadza

bunter-gatherers compared with urban Italians. symptoms in the mice, would we see changes in their behavior?"

Mazmanian and Hsiao investigated by dosing the animals with a microbe known for its anti-inflammatory properties, Bacteroides fragilis, which also protects mice from experimentally induced colitis. Results showed that the treatment fixed intestinal leaks and restored a more normal microbiota. It also mitigated the tendency toward repetitive behavior and reduced communication. Mazmanian subsequently found that $B$. fragilis reverses MIA deficits even in adult mice. "So, at least in this mouse model, it suggests features of autism aren't hardwired — they're reversible — and that's a huge advance," he says.

\section{LIMITS OF RESEARCH}

THE HUMAN GUT MICROBIOME evolved to help us in myriad ways: Gut microbes make vitamins, break dietary fiber into digestible short-chain fatty acids and govern normal functions in the immune system. Probiotic treatments such as yogurt supplemented with beneficial strains of bacteria are already being used to help treat some gastrointestinal disorders, such as antibiotic-induced diarrhea. But there are little data about probiotic effects on the human brain.

In a proof-of-concept study Mayer and his colleagues at U.C.L.A. uncovered the first evidence that probiotics ingested in food can alter human brain function. The researchers gave healthy women yogurt twice a day for a month. Then brain scans using functional magnetic resonance imaging were taken as the women were shown pictures of actors with frightened or angry facial expressions. Normally, such images trigger increased activity in emotion-processing areas of the brain that leap into action when someone is in a state of heightened alert. Anxious people may be uniquely sensitive to these visceral reactions. But the women on the yogurt diet exhibited a less "reflexive" response, "which shows that bacteria in our intestines really do affect how we interpret the world," says gastroenterologist Kirsten Tillisch, the study's principal investigator. Mayer cautions that the results are rudimentary. "We simply don't know yet if probiotics will help with human anxiety," he says. "But our research is moving in that direction."

Strains of Bifidobacterium, which is common in the gut flora of many mammals, including humans, have generated the best results so far. Cryan recently published a study in which two varieties of Bifidobacterium produced by his lab were more effective than escitalopram (Lexapro) at treating anxious and depressive behavior in a lab mouse strain known for pathological anxiety. Although Cryan is optimistic that such findings may point the way to the development of psychobiotics, he is wary of hype. "We still need a lot more research into the mechanisms by which gut bacteria interact with the brain," he says.

Charles Schmidt is a recipient of the National Association of Science Writers' Science in Society Journalism Award. His work has appeared in Science, Nature Biotechnology, Nature Medicine and the Washington Post. 